\title{
Inactivation of sortilin (a novel lysosomal sorting receptor) by dominant negative competition and RNA interference
}

\author{
Stephane Lefrancois ${ }^{1 *}$, Maryssa Canuel ${ }^{1}$, Jibin Zeng ${ }^{1}$ and Carlos R. Morales ${ }^{1}$
}

\author{
${ }^{1}$ Department of Anatomy and Cell Biology, McGill University, Montreal, Quebec, Canada. \\ *Corresponding Author: Stephane Lefrancois, Cell Biology and Metabolism Branch, National Institute of Child Health and Human Development, National Institutes \\ of Health, 9000 Rockville Pike, Building 18T, Room 101, Bethesda, MD 20892. Phone: (301) 402-3610; Fax: (301) 402-0078; Email: lefrancs@mail.nih.gov
}

Submitted: October 25, 2004; Revised: January 10, 2005; Accepted: January 10, 2005.

Indexing terms: Sortilin; Sphingolipid Activator Proteins.

Abbreviations: $\mathrm{GM}_{2} \mathrm{AP}, \mathrm{GM}_{2}$ activator protein; LAMP, lysosomal associated protein; LIMP, lysosomal integral membrane protein-1; M6P-R, mannose 6phosphate receptor; RNAi, RNA interference; SAP, sphingolipid activator protein; siRNA, small interference RNA; shRNA, small hairpin interference RNA; AP-1, Adaptor Protein-1.

\begin{abstract}
To assess the role of sortilin in the sorting and trafficking of sphingolipid activator proteins (SAPs) the function of sortilin was abolished by a dominant-negative mutant and by the use of RNAi. Mutant sortilin lacking the carboxylterminal region that contains the sorting signal abolished the trafficking of SAPs to the lysosomes. Both sortilin and SAPs were retained in the Golgi apparatus. The use of chemically synthesized siRNA effectively blocked the trafficking of SAPs to the lysosomes as well. Additionally, we created a stable COS-7 cell line transfected with the pSilencer $3.1 \mathrm{H} 1$ neo vector containing a selected siRNA template oligonucleotide (small hairpin interference RNA) where the levels of sortilin were greatly suppressed. The elimination of sortilin by this method will permit to determine whether or not sortilin is involved in a general mechanism of lysosomal sorting that involves the trafficking of various soluble lysosomal proteins other than SAPs.
\end{abstract}

\section{INTRODUCTION}

Protein trafficking in eukaryotic cells involves a series of protein-protein interactions between the cargo and the proteins that form the transport carrier. Cargo proteins are selected for sorting to specific destinations by coat proteins or by receptors that in turn interact with coat proteins (1). Various organelles have a repertoire of receptors and coat proteins that specify the final destination of the cargo protein. The coat proteins cause trafficking vesicles to bud from donor membranes and to traffic to acceptor membranes. Major sites of vesicle formation and budding include the endoplasmic reticulum, the plasma membrane and the Golgi apparatus. The Golgi apparatus is the site of sorting for newly synthesized proteins destined to the lysosomal compartment. Two different types of proteins are sorted to the lysosomes, transmembrane and soluble lysosomal proteins. Due to their specific characteristics these two types of proteins use different sorting mechanisms.

Lysosomal membrane proteins such as LAMPs and LIMPs interact directly with the coat-proteins, AP-1 and AP-3. These 2 complexes are members of the multimeric adaptor complexes that also include AP-2 and AP-4. Each of these complexes is composed of a large subunit $(\beta 1-4)$, a medium subunit $(\mu 1-4)$, a small subunit $(\sigma 1-4)$ and a non-homologous subunit $(\gamma, \alpha, \delta, \varepsilon)$ (2). Coat proteins interact with lysosomal membrane proteins at two different sites. Tyrosine based motifs (YXXФ, where $\mathrm{X}$ is any amino acid and $\Phi$ is a bulky hydrophobic amino acid) from proteins such as LAMP-1 and CD63 bind to AP- 1 and AP-3 via their $\mu 1$ and $\mu 3$ subunits respectively while dileucine motifs (DXXLL) from proteins such as 
tyrosinase and LIMP-II bind to $\gamma-\sigma 1$ and $\delta-\sigma 3$ hemicomplexes (3). Disruption of these interactions causes missorting of the cargo to the plasma membrane.

The Golgi apparatus has a second set of coat proteins known as Golgi-localized, year-containing ARF-binding proteins (GGAs). The GGAs are three homologous monomeric adaptor proteins (4). While the multimeric adaptors are directly recruited to the Golgi membrane by the transmembrane proteins LAMPs, LIMPS and tyrosinase, the monomeric adaptors bind to transmembrane receptors such as the mannose 6phosphate receptor (M6P-R) (4) and sortilin (5). Interestingly, both receptors bind soluble lysosomal proteins such as cathepsins and various other soluble hydrolases in the case of the M6P-R and sphingolipid activator proteins (prosaposin and $\mathrm{GM}_{2} \mathrm{AP}$ ) in the case of sortilin (6). Several reports have demonstrated that disruption of GGAs interferes with the sorting of soluble cargos to the lysosomal compartment $(4,7)$.

Sphingolipid Activator Proteins (SAPs) are a group of five lysosomal proteins involved in the catabolism of lipids in the lysosome $(8,9)$. Four of the proteins, the saposins, originate from the lysosomal cleavage of the common precursor protein prosaposin (10). The fifth SAP is the $\mathrm{GM}_{2}$ activator protein (11). The five SAPs are effectors of the enzymes that breakdown glycosphingolipids. We have recently reported that prosaposin and $\mathrm{GM}_{2}$ activator protein require sortilin to traffic to the lysosomal compartment by using dominant negative competitors and RNAi technology to suppress sortilin and GGAs (6). In the present paper, we discuss the use of these dominant negative proteins and RNAi, their potential benefits and disadvantages in the study of protein trafficking to the lysosomes.

\section{MATERIALS AND METHODS}

\section{Rationale}

Previous reports demonstrated that sortilin could bind GGAs via its cytoplasmic tail. This suggested a possible role for sortilin in the transport of proteins to the lysosomal compartment. Paradoxically, no cargo had been demonstrated to bind or use this potential sorting receptor. Since SAPs had been shown to reach the lysosomes in an M6P-R independent manner, we hypothesized that sortilin was the sorting receptor for SAPs. To test this hypothesis, we developed and used a dominant negative form of sortilin, which lacked the GGA binding motif. Additionally, we developed a set of siRNA probes to knockdown sortilin expression in COS7 cells and we examined the effect of sortilin inactivation on the transport of SAPs. In this paper we are extending our RNAi results by including a plasmid-based expression approach that produces hairpin RNA species capable of preventing the synthesis of sortilin. This new approach appears to be more effective since the expression plasmid produces short hairpin RNA molecules constitutively and allows for selection of RNAi positive clones.

\section{Dominant negative procedure}

A useful tool to investigate the function of a targeting protein is to introduce into the cell a mutant version to compete with the endogenous version of the protein and cause a malfunction in the system. To test whether or not sortilin was the sorting receptor for SAPs, we constructed a mutant protein of sortilin that lacked its sorting signal in the cytoplasmic tail without affecting its capacity to bind SAPs in the luminal compartment of the Golgi apparatus. We expected this mutant protein to be retained in the Golgi apparatus or to be missorted to the plasma membrane. Additionally, we expected to observe distinct biochemical and immunostaining patterns for SAPs due to the inability of the sorting receptor to traffic to the lysosomes.

The mutant protein was designed using primers to eliminate the carboxy-terminal tail of sortilin, which contains the lysosomal sorting signal. Since this assay is based on the competition of truncated versus endogenous sortilin the success of this experiment depended upon the higher level of expression of the recombinant protein. Another requirement for this experiment was the introduction of an epitope (myc-tag) that allows distinguishing the recombinant from endogenous sortilin. To meet these requirements the truncated protein was cloned into the pcDNA3.1 vector, a mammalian expression vector. Since this vector is designed to add a c-terminal myc-tag to the cloned protein, we were able to distinguish mutant sortilin in COS-7 cells and in western blot analysis with an antimyc antibody (Invitrogen, Burlington, $\mathrm{ON}$ ). To examine 
the localization of mutant sortilin, we transiently transfected COS-7 cells with the pcDNA3.1 vector using a DEAE/Dextran chloroquine phosphate technique. Cells were then incubated for 48 hours to allow the mutant protein to be expressed. The dominant negative form of sortilin lacking its sorting tail was detected by immunofluorescence confocal microscopy and western blot analysis.

\section{RNAi procedures}

siRNA: Designing a small interfering RNA (siRNA) is an important step for successful knockdown of a target protein. One potential obstacle is the fact that the design is almost random, in that no specific sequence is guaranteed to knockdown the targeted mRNA. Several companies, including Ambion (Austin, TX) and Dharmacon (Lafayette, CO), provide algorithms to assist in the design of siRNAs. The key requirement of this approach is to select several sequences of a protein that are not found in any other proteins searched in Blast. This allows the design of specific siRNAs that will only target the desired protein. Although this is a clear advantage of siRNA, a recent report suggests that the specificity achieved by this procedure may not be as high as initially thought (12). As well, the starting requirements for selecting an siRNA sequence include a sequence beginning with an AA di-nucleotide, having no stretches of greater than four $T^{\prime}$ s, and a $G / C$ content between $30-50 \%$ (13). After selecting the specific sequences from the target protein, the siRNAs may be synthesized using commercial kits or via a commercial laboratory. Although this procedure is much less expensive than producing a mouse $\mathrm{KO}$, sometimes the generation of siRNAs can prove to be costly. Since commercial siRNAs can range from 200-500 U.S. dollars per sample the use of this service could be a good alternative if the selected sequence is already known to work. siRNAs can be ordered from Qiagen (Mississauga, $\mathrm{ON}$ ) and Dharmacon (Lafayette, CO) or other laboratories. In our case we produced our own siRNAs in situ using a kit from Ambion (Austin, TX). Thus, for the same price is it is possible to generate up to 12 different siRNAs.

Once the siRNA is produced it must be transiently transfected into a mammalian cell line. Several methods are available for transfecting culture cells with either plasmids that transcribe RNAi or siRNA directly. Roche (Laval, QC) and Invitrogen (Burlington, ON) have available liposome-based systems. In fact, Invitrogen developed a transfection reagent specific for oligo siRNAs called Oligofectamine. These products work well and are used extensively although cost can be a factor in some cases. In our hands, a simple DEAEdextran/Chloroquine phosphate technique worked optimally. According to this procedure COS-7 cells are plated overnight in serum free media and transfected with the recommended amount of siRNA. The cells are placed at $37^{\circ} \mathrm{C}$ for 48 hours. In our experience, incubation times may vary greatly. In some cases, two doses of siRNA transfection are required, with an interval of 48 or 72 hours. We normally incubate our cells for a total of six days.

Hairpin RNAi: The hairpin consists of a single-stranded RNA containing two regions of complementary sequences that refold into a double-stranded loop region and a connecting loop stem with a $3^{\prime}$ terminal uridine tract. Based on our siRNA results we selected an internal sortilin sequence (AAGGTGGTGTTAACAGCAGAG) for designing the short hairpin RNA (shRNA). The sequence was subcloned into the plasmid-based expression vector pSilencer 3.1 H1-neo that contains a strong H1 RNA pol III promoter (Ambion, Austin, TX). We used Lipofectamine 2000 (Invitrogen, Burlington, ON) as described by the manufacturer to transfect the plasmid and selected for resistant cells to neomycin (GIBCO, Burlington, $\mathrm{ON}$ ), which expressed constitutively the shRNA. The purpose of this strategy is to initiate the production of shRNA to trigger dicer cleavage and led to the sortilin mRNA silencing.

\section{RESULTS AND DISCUSSION}

\section{Effect of dominant negative sortilin}

The objective of this experiment was to introduce a dominant negative mutant sortilin in COS-7 cells to compete with endogenous sortilin and cause the retention and/or missorting of their putative ligands, i.e., sphingolipid activator proteins (SAPs). This technique has been used extensively to abolish the function of signaling proteins (14). In this case we constructed a mutant sortilin that lacked the cytoplasmic sorting signal, thereby retaining the receptor in the Golgi or missorting it to the plasma membrane. We hypothesized that if sortilin was the receptor for SAPs, the lysosomal localization of these proteins should also change with the localization of the mutant protein. Using an anti-myc antibody, our results indicated a steady state production 
and retention of mutant sortilin in the Golgi apparatus, as shown by its co-localization staining with the antiGolgin antibody (Figs. 1.1-1.3). Since the mutant protein was retained in the Golgi apparatus, we predicted that the cargo of this receptor should remain in the same compartment and/or be missorted to the extracellular space. To examine the effect of the dominant-negative construct on the lysosomal trafficking of SAPs, COS-7 cells were immunostained with either anti-prosaposin (Figs. 1.2-1.3) or anti-GM2AP antibodies (6) after transient transfection with the mutant sortilin. Control COS-7 cells stained for SAPs showed punctate structures that colocalized with LysoTracker or other lysosomal markers such as cathepsin B and LAMP-2 (6). Cells expressing mutant sortilin exhibited little or no punctate staining. This was interpreted as a demonstration of reduced amounts of the SAPs in the lysosomal compartment as compared to the non-transfected cells. On the other hand, mutant sortilin had no effect on the lysosomal localization of cathepsin B, a protein that requires the M6P-R to traffic to lysosomes (6).

\section{Effect of sortilin siRNAs}

Using the Ambion kit we synthesized six siRNAs against sortilin. To determine the effect of the siRNAs we examined both mRNA and protein levels using RT-PCR and Western blotting and/or immunofluorescence. First, we isolated mRNA from transfected and non-transfected (control) cells using a Qiagen's Rneasy kit (Mississauga, ON), and performed RT-PCR. Our results showed that siRNA 1 (AGGTGGTGTTAACAGCAGAG) and siRNA 3 (AATGTTCCAATGCCCCACTC) successfully inhibited the expression of sortilin mRNA in COS-7 cells. To verify that transfection did not inhibit the expression of unrelated proteins we examined the level of $\beta$-actin mRNA. $\beta$-Actin is expressed constitutively in most cells. The levels of $\beta$-actin transcripts were measured by RTPCR in normalized samples of transfected or nontransfected cells. Our results showed that $\beta$-actin transcripts were not affected by the siRNA treatment (6). Cell lysates were simultaneously prepared from COS-7 cells transfected or not with sortilin siRNAs and run on an SDS-PAGE gel. Immunoblotting confirmed that siRNAs 1 and 3 were most effective in reducing the amount of sortilin, but not $\beta$-actin in transfected cells. To verify the immunoblotting results, we evaluated the level of sortilin in these cells using immunofluorescence and confocal microscopy. Confocal microscopy demonstrated that sortilin siRNA abolished the immunostaining of the anti-sortilin antibody (Figs. 1.4-1.6).
After confirming the inhibitory effect of the sortilin siRNA we tested the hypothesis that sortilin is the sorting receptor for SAPs. To this effect we used siRNA (sequence 1) which knockdown sortilin most efficiently in COS-7. We predicted that knocking down sortilin would affect the localization of SAPs. To assess this prediction we immunostained COS-7 cells treated with sortilin siRNA with anti-prosaposin, anti-GM2AP and anti-cathepsin B antibodies. Therefore, cargo that requires sortilin to be sorted to the lysosomes should be absent from or exhibit reduced immunostaining in the punctate structures. Conversely, cargo that did not depend on sortilin should continue to be visible in punctate structures. As predicted, prosaposin (Fig. 1.5) and $\mathrm{GM}_{2} \mathrm{AP}$ were absent from punctate structures of siRNA treated cells, while cathepsin B was not confirming a role for sortilin in the trafficking of SAPs to the lysosomal compartment (6).

\section{Use of hairpin RNAi}

Inactivation of genes with conventionally synthesized siRNAS may encounter several problems such as relatively high background levels produced by cells that do not incorporate the siRNA probe. Similarly, time constraints due to the transient nature of this silencing approach may affect the outcome of some experiments $(15,16)$. A recent technology that represents an improvement to these problems is the use of plasmids that express functional siRNAs for long-term studies (17). Since plasmids contain antibiotic resistant genes it is also possible to select successfully transfected cells and therefore to significantly lower the background produced by non-transfected cells (17).

Using this approach we have created a COS-7 cell line transfected with the pSilencer 3.1 H1-neo vector from Ambion that contained a subcloned sortilin siRNA template oligonucleotide (sequence 1). The subcloned sequence included two complementary regions that permit the refolding of the transcribed RNA into a double-stranded region and a connecting stem (shRNA). The production of shRNA triggers Dicer cleavage and leads to the silencing of sortilin mRNA. To verify the expression of the shRNA and the knockdown of sortilin, RT-PCR and Western blotting of sortilin were conducted as for the in vitro siRNA experiments. Both analyses showed that the levels sortilin expression was decreased. These results were further confirmed by immunofluorescence and confocal microscopy (Figs. 1.71.8). We expect to employ this sortilin deficient COS-7 
cell line for proteomic analyses to determine if other

lysosomes bound to sortilin. soluble lysosomal proteins other than SAPs traffic to the
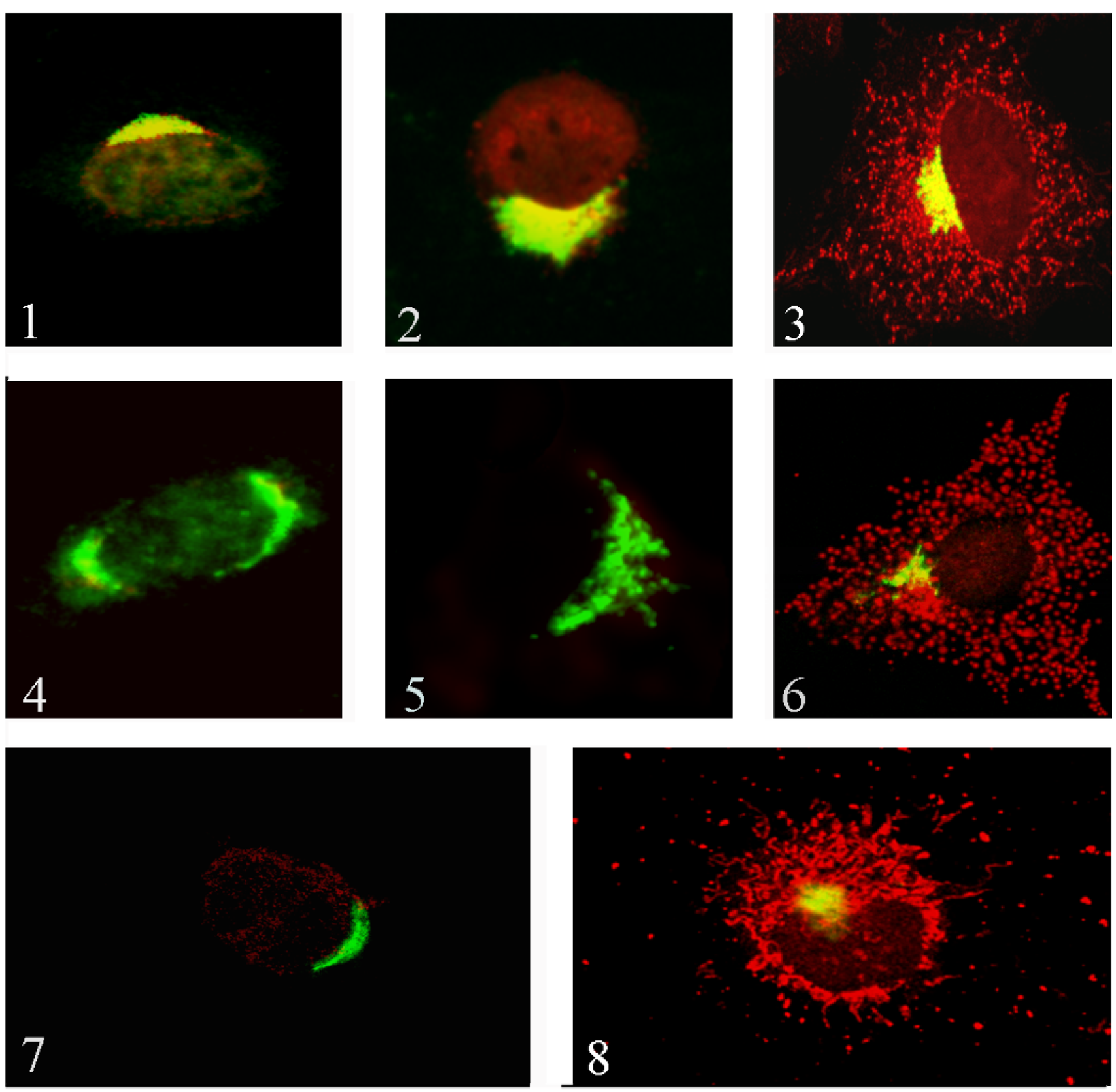

Fig. 1.1: coS-7 cell transfected with dominant-negative (truncated) sortilin fused to a myc-tag protein. Anti-myc antibody (green) colocalizes with antiGolgin antibody (red) in the perinuclear region of the cell. The overlay of both reactions yields yellow color indicating that truncated sortilin is retained in the Golgi apparatus. Magnification: x400. Fig. 1.2: COS-7 cell transfected with dominant-negative (truncated) sortilin fused to a myc-tag protein. Anti-myc antibody (green) colocalizes with anti-prosaposin antibody (red) in the perinuclear region of the cell. The overlay of both reactions yields yellow color indicating that prosaposin and sortilin were retained together in the Golgi apparatus. Magnification: x400. Fig. 1.3: CoS-7 cell transfected with wild type sortilin construct (control) and immunostained with anti-Golgin (green) and anti-prosaposin (red) antibodies. The overlay shows yellow color in the perinuclear region and red granular in the cytoplasm typical of lysosomal staining. Magnification: $x 400$. Fig. 1.4: COS-7 cell transfected with sortilin siRNA probe (sequence 1). The siRNA abolished the perinuclear staining of sortilin antibody (red). The overlay shows green fluorenscence only produced by the anti-Golgin antibody. Magnification: x400. Fig. 1.5: Cell transfected with a sortilin siRNA probe. The cell was stained with anti-sortilin (red) and anti-prosaposin (green) antibodies. The siRNA abolished the staining of sortilin and the punctate staining of the prosaposin antibody. Note that prosaposin is retained in the Golgi apparatus (green). Magnification: x400. Fig. 1.6: Control CoS-7 cell (non transfected with siRNA) immunostained with anti-Golgin (green) and anti-prosaposin (red) antibodies. The red granular reaction is typical of prosaposin lysosomal staining. Magnification: x400. Fig. 1.7: COS-7 cell transfected with sortilin short hairpin interference RNA (shRNA) vector (sequence 1). The shRNA abolished the perinuclear staining of sortilin antibody (red). The overlay shows green fluorescence only, produced by the anti-Golgin antibody. Magnification: $x 400$. Fig. 1.8: COS-7 cell transfected with empty vector (control) immunostained with anti-Golgin (green) and anti-prosaposin (red) antibodies. The red granular reaction is typical of prosaposin lysosomal staining. Magnification: $x 400$. 


\section{CONCLUSIONS}

To study lysosomal trafficking pathways in living cells, several groups have taken advantage of mutant proteins and RNAi to disclose the role of proteins involved in these processes. Although both methods provide useful information on the function of sorting receptors, they also present some limitations. The introduction of mutant proteins as dominant-negative competitors into the cell is an excellent way of abolishing the function of a protein. In our hands, mutant sortilin lacking its carboxyl-terminal region demonstrated that sortilin is involved in the trafficking of the SAPs to the lysosomal compartment. However, the effect of mutant protein on other cellular functions should be a concern. For that matter it is important to examine control proteins with closely related cellular functions such as a protein or proteins involved in alternative trafficking pathways. Ideally, an alternative pathway that involves a different receptor should not be affected by the mutant protein.

RNAi technology has also become a powerful tool in the investigation of gene function. This approach has made it possible to identify the role of novel genes much quicker than in the past. In our hands, we successfully determined the function of sortilin, a protein that interacts with coat proteins via its cytoplasmic domain but whose cargo remained largely unidentified. Knocking down sortilin interfered with the trafficking of SAPs to the lysosomes, suggesting a role for sortilin in the sorting and trafficking of SAPs to the lysosomal compartment.

Although RNAi technology is useful, this method also presents some limitations. siRNAs used in cells offer a specific mechanism to target mRNA and knockdown a specific protein. However, the process is not as specific as initially thought. Short RNA inserts are required to prevent the cell from going through a viral response much like it would if being infected by a double stranded RNA virus (15). However, recent evidence suggests that the short RNA may cause some of the viral response to occur anyway. In addition, the specificity of the probe could be often in question. Although the probe is designed to bind to a single sequence, it is possible that it binds other sequences in a non-specific manner resulting in the knockdown of undesired proteins. This problem could be difficult to address since it is almost impossible to test the levels of all proteins in the cell. The best way to avoid both problems is to reduce the amount of siRNA to a minimum in the experiment. In practical terms, using low amounts of siRNA reduces the costs and increases the specificity of the experiment by avoiding non-specific knockdown of unrelated proteins and preventing the cell from undergoing a viral response. Although no specific quantities of siRNA will work for a given experiment, amounts in the range of $0.02 \mu \mathrm{M}$ have worked well. In any case, each siRNA must be considered differently and should be tested at different concentrations.

Finally, the combination of two or more approaches is the safest way to overcome the deficiency of a single method. In our case the use of dominant-negative mutants and siRNA have allowed us to characterize the role of sortilin in the sorting of SAPs. However, it is possible that sortilin is also involved in the sorting of other soluble lysosomal proteins. Currently, it is well accepted that soluble hydrolases must bind to the mannose-6-phosphate receptor (M6P-R) to exit from the Golgi apparatus and in fact M6P-R is considered the main lysosomal sorting receptor. Nevertheless, a number of laboratories have demonstrated that the lysosomes of I-cell disease (ICD) fibroblasts, resulting from a mutation in the phosphotransferase that adds mannose-6phosphate to hydrolases, have near normal levels of some lysosomal hydrolases, in addition, to the sphingolipid activator proteins prosaposin and $\mathrm{GM}_{2} \mathrm{AP}$ (16). This evidence suggests the existence of an alternative pathway. It is then possible that sortilin is involved in the targeting of hydrolases that do not use the M6P-R. This is the main focus of our laboratory. Consequently, we are pursuing the identification of all soluble hydrolases that use this alternative receptor by means of RNAi and proteomics. Our study will permit to identify other bona fide sortilin ligands and to predict the consequences of sortilin mutations. Nonetheless, the transient nature of siRNA technology, the relatively high levels of background and the time constraints due to the transient nature of silencing are problems that may affect the outcome of our experiments. Therefore, we have created a stable COS-7 cell line transfected with the pSilencer 3.1 H1 neo vector (Ambion) containing a selected siRNA template oligonucleotide (small hairpin interference RNA). As desired the levels of sortilin have been greatly suppressed. We intend to analyze the effect 
of the elimination of sortilin using a proteomics approach. Immunochemically we shall verify the absence of cargo in the lysosomes of the established cell line.

In conclusion, by using dominant-negative mutants and RNAi technology we were able to characterize a novel lysosomal sorting receptor (sortilin) involved in the alternative sorting of SAPs (prosaposin and $\mathrm{GM}_{2} \mathrm{AP}$ ). The improvement of RNAi in conjunction with proteomics and other approaches should allow us to characterize the effects of sortilin inactivation on the sorting of soluble lysosomal proteins other than SAPs and to determine if sortilin is responsible of a more general mechanism of lysosomal sorting.

\section{ACKNOWLEDGMENTS}

The authors are grateful to Jacob Hassan for providing the confocal microscope images of the sortilin dominantnegative and sortilin siRNA. Stephane Lefrancois is the recipient of a post-doctoral fellowship from CIHR. This work was supported by a CIHR grant to Carlos R. Morales.

\section{REFERENCES}

1. Bonifacino JS, Lippincott-Schwartz J. Coat proteins: shaping membrane transport. Nat Rev Mol Cell Biol 2003; 4:409-414.

2. Robinson MS, Bonifacino JS. Adaptor-related proteins. Curr Opin Cell Biol 2001; 13:444-453.

3. Janvier K, Kato Y, Boehm M, Rose JR, Martina J, Kim BY, Venkatesan S, Bonifacino JS. Recognition of dileucine-based sorting signals from HIV-1 Nef and LIMP-II by the AP- $1 \quad \gamma-\sigma 1$ and AP-3 $\delta-\sigma 3$ hemicomplexes. Journal of Cell Biology 2003; 163:12811290.

4. Puertollano R, Aguilar RC, Gorshkova I, Crouch RJ, Bonifacino JS. Sorting of mannose 6-phosphate receptors mediated by the GGAs. Science 2001; 292:1712-1716.

5. Nielsen MS, Madsen P, Christensen EI, Nykjaer A, Gliemann J, Kasper D, Pohlmann R, Petersen CM. The sortilin cytoplasmic tail conveys Golgiendosome transport and binds the VHS domain of the GGA2 sorting protein. Embo J 2001; 20:2180-2190.

6. Lefrancois S, Zeng J, Hassan AJ, Canuel M, Morales CR. The lysosomal trafficking of sphingolipid activator proteins (SAPs) is mediated by sortilin. Embo J 2003; 22:6430-6437.

7. Zhu Y, Doray B, Poussu A, Lehto VP, Kornfeld S. Binding of GGA2 to the lysosomal enzyme sorting motif of the mannose 6-phosphate receptor. Science 2001; 292:1716-1718.

8. Kishimoto Y, Hiraiwa M, O'Brien JS. Saposins: structure, function, distribution, and molecular genetics. J Lipid Res 1992; 33:1255-1267.

9. Mahuran DJ. The GM2 activator protein, its roles as a co-factor in GM2 hydrolysis and as a general glycolipid transport protein. Biochim Biophys Acta 1998; 1393:1-18.

10. Hiraiwa M, Martin BM, Kishimoto Y, Conner GE, Tsuji S, O'Brien JS. Lysosomal proteolysis of prosaposin, the precursor of saposins (sphingolipid activator proteins): its mechanism and inhibition by ganglioside. Arch Biochem Biophys 1997; 341:17-24.

11. Swallow DM, Islam I, Fox MF, Povey S, Klima H, Schepers U, Sandhoff K. Regional localization of the gene coding for the GM2 activator protein (GM2A) to chromosome 5q32-33 and confirmation of the assignment of GM2AP to chromosome 3. Ann Hum Genet 1993; 57:187-193.

12. Jackson AL, Bartz SR, Schelter J, Kobayashi SV, Burchard J, Mao M, Li B, Cavet G, Linsley PS. Expression profiling reveals off-target gene regulation by RNAi. Nat Biotechnol 2003; 21:635-637.

13. Elbashir SM, Harborth J, Lendeckel W, Yalcin A, Weber K, Tuschl T. Duplexes of 21-nucleotide RNAs mediate RNA interference in cultured mammalian cells. Nature 2001; 411:494-498.

14. Feig LA. Tools of the trade: use of dominantinhibitory mutants of Ras-family GTPases. Nat Cell Biol 1999; 1:E25-E27.

15. Berns K, Hijmans EM, Mullenders J, Brummelkamp TR, Velds A, Heimerikx M, Kerkhoven RM, Madiredjo M, Nijkamp W, Weigelt B, Agami R, Ge W, Cavet G, Linsley PS, Beijersbergen RL, Bernards R. A large-scale RNAi screen in human cells identifies new components of the p53 pathway. Nature 2004; 428:431-437.

16. Paddison PJ, Hannon GJ. RNA interference: the new somatic cell genetics? Cancer Cell 2002; 2:17-23.

17. Brown D, Jarvis R, Pallota V, Byrom M, Ford L. RNA interference in mammalian cell culture: design, execution, and analysis of the siRNA effect. Ambion Tech Notes 2002; 9:3-5. 
18. Sledz CA, Holko M, de Veer MJ, Silverman RH, Williams BR. Activation of the interferon system by short-interfering RNAs. Nat Cell Biol 2003; 5:834-839.
19. Morimoto S, Yamamoto Y, O'Brien JS, Kishimoto Y. Distribution of saposin proteins (sphingolipid activator proteins) in lysosomal storage and other diseases. Proc Natl Acad Sci USA 1990; 87:3493-3497. 


\section{PROTOCOLS}

\section{DEAE/Dextran chloroquine phosphate method for $100 \mathrm{~mm}$ plates}

Day 1: Plate out cells in serum free medium $\left(5 \times 10^{5}\right.$ per $100 \mathrm{~mm}$ culture plate) and incubate overnight

Day 2: Prepare the following solutions before proceeding:

- $10 \%$ DMSO in sterile PBS

- $250 \mathrm{mg}$ chloroquine phosphate in $5 \mathrm{ml}$ serum free medium

- $250 \mathrm{mg}$ DEAE/dextran in $5 \mathrm{ml}$

1. Mix $50 \mu \mathrm{l}$ of DEAE/dextran and $10 \mu \mathrm{l}$ of chloroquine phosphate into $10 \mathrm{ml}$ of serum free media. Add $25 \mu \mathrm{g}$ of DNA.

2. Aspirate medium from cells and wash with sterile PBS or medium to remove dead cells

3. Replace with transfection solution mixed previously and incubate for 4 hours at $37^{\circ} \mathrm{C}$

4. After 4 hour incubation, shock cells with $10 \%$ DMSO for 1 minute per plate

5. Wash plates with sterile PBS twice and replace with fresh DMEM containing fetal calf serum and antibiotics

6. Incubate for $48-72$ hours

Day 4 or 5: Analyze cells via immunostaining and Western blotting

\section{Hairpin RNAi}

siRNA template sequences can be sent to the Descript Corporation (Piscataway, NJ, USA) in order to be cloned into the pSilencer vector from Ambion, or cloned in your lab into the vector as follows:

1. Annealing. Reaction must be prepared according to Ambion protocol. Incubate the reagents at $90^{\circ} \mathrm{C}$ for 3 minutes and then cool the preparation to $37^{\circ} \mathrm{C}$ in a water bath for 1 hour.

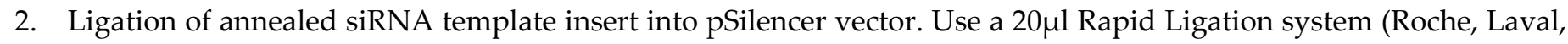
QC) according to the manufacturer's instructions. A 3:1 ratio of insert to vector must be used.

3. Amplification of the ligated pSilencer vector. Use $2 \mu \mathrm{l}$ of pSilencer with the siRNA template and a pSilencer control vector with no insert to transform DH $\alpha 5$ E. coli (Bio S\&T, Montreal, QC) according to manufacturer's protocol onto LB plates containing $50 \mu \mathrm{g} / \mathrm{ml}$ of ampicillin. Plates where the plus-insert ligation yield 5-10 fold more colonies than the minus-insert ligation must be used to harvest 4-6 colonies from which vector is purified according to specifications of a Qiagen HiSpeed Plasmid Midi Purification Kit.

4. Identification of clones with the hairpin siRNA insert. Digest $25 \mu \mathrm{g}$ of plasmid with BamH1 and HindIII restriction enzymes according to Sigma's protocol (St. Louis, MO, USA).

5. Digestion products must be analyzed on a 10\% PAGE gel. Visualize siRNA template insert (66bp and a $4486 \mathrm{~kb}$ vector band).

6. Identification of positive clones must be confirmed by sequencing.

\section{Transfection into COS-7 cells}

1. Plate $0.5 \times 10^{5}$ cells in a 24 well plate and transfect them with Lipofectamine 2000 as described by Invitrogen. Mix $0.9 \mu \mathrm{g}$ of DNA and $2 \mu \mathrm{l}$ of Lipofectamine in serum-free DMEM medium (GIBCO). After 24 hours post-transfection, replace the growth medium with serum-containing medium.

2. Two days subsequent to transfection cells should be transferred to a 6-well plate and siRNA positive cells must be selected with $0.75 \mathrm{mg} / \mathrm{ml}$ of neomycin for 10-13 days.

3. The efficiency of the knockdown must be assessed by RT-PCR and Western blotting. 